\title{
Modeling of Piezoelectric Actuators Based on a New Rate-Independent Hysteresis Model
}

\author{
Jingyang Peng, Xiongbiao Chen \\ Department of Mechanical Engineering, University of Saskatchewan, Saskatoon, Canada \\ E-mail:jip747@mail.usask.ca \\ Received September 27, 2011; revised October 28, 2011; accepted November 5, 2011
}

\begin{abstract}
Accurate model representatives of piezoelectric actuators (PEAs) are important for both understanding the dynamic behaviors of PEAs and control scheme development. However, among the existing models, the most widely used classical Preisach hysteresis model are incapable of representing the commonly-encountered one-sided (non-negative voltage input range) hysteresis behaviors of PEAs. To solve this problem, a new rate-independent hysteresis model was developed for the one-sided hysteresis and then integrated with the models representative of creep and dynamics to form a single model for the PEAs. Experiments were carried out to validate the developed models.
\end{abstract}

Keywords: Piezoelectric Actuator, Hysteresis

\section{Introduction}

Piezoelectric actuators (PEAs) have been widely used in micro-/nano-positioning systems due to their fine displacement resolution and large actuation force [1]. In such applications, accurate models of PEAs are usually required for both understanding of their dynamic behaviors and controller design. A widely-used category of PEA models takes the form of a cascade of three sub-models, each of which representing the effect of hysteresis, creep, and vibration dynamics, respectively, e.g. [2]. While the modeling of the vibration dynamics and creep has been well addressed in the literature, there are still problems with the modeling of hysteresis. Most commercially available PEAs have a non-negative input voltage range and their corresponding hysteresis behaviors subject to such one-sided input range are referred to as one-sided hysteresis, as shown in Figure 1, which contains an initial ascending curve in addition to the hysteresis loops. It is observed in the authors' earlier study [3] that the classical Preisach (CP) hysteresis model [4] cannot represent such one-sided hysteresis since it can not represent the initial ascending curve. This deficiency of the CP hysteresis model has been neglected in the literature, e.g. [5-8]. This problem of modeling the one-sided hysteresis was solved in [3] by developing a new rate-independent (RI) hysteresis model based on a novel hysteresis operator.

On this basis, in this paper, this new RI hysteresis model

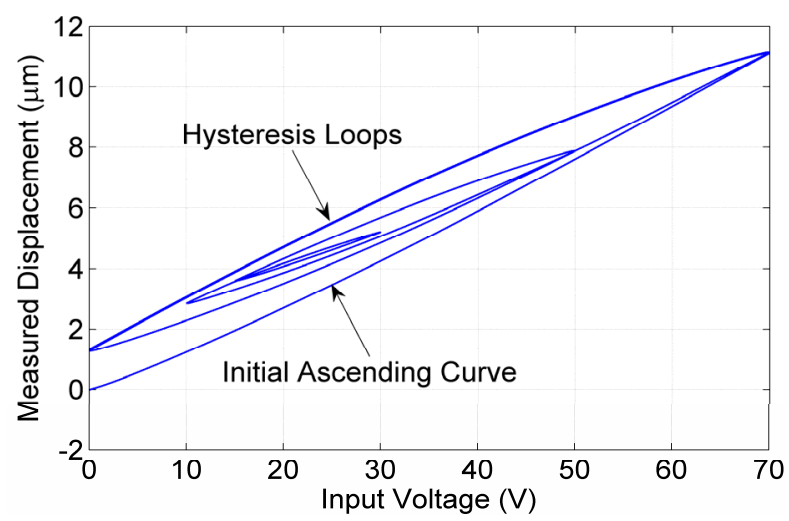

Figure 1. One-sided hysteresis behavior measured from a PEA subjected to non-negative voltage input.

is integrated with a vibration sub-model and a creep submodel to form an integrated model for PEAs. The parameter estimation scheme for such a model of PEAs is also developed. Experiments were conducted and the results obtained were compared with simulation results to validate the model developed.

\section{Outline of the Rate-Independent (RI) Hysteresis Model}

The RI hysteresis model developed in [3] represents onesided hysteresis behaviors as the combined effects of an infinite number of hysteresis operators, one of which is 
shown in Figure 2. Compared to the Preisach hysteresis operator, the hysteresis operator in Figure 2 has one more lower saturation value to account for the initial ascending curve in one-sided hysteresis. The two switching values satisfy $\alpha \geq \beta$. The hysteresis is then expressed mathematically as

$$
f(t)=\iint_{u_{\max } \geq \alpha \geq \beta \geq u_{\min } \geq 0} \delta(\alpha, \beta, u(t)) \mathrm{d} \alpha \mathrm{d} \beta
$$

where $f(t)$ is the hysteresis, i.e. the model output, $u_{\max }$ and $u_{\min }$ are the maximum and minimum input $u(t)$ in history, respectively [3].

The double integration suggests that the hysteresis model output is the combined effect of an infinite number of hysteresis operators with bounded values of $\alpha$ and $\beta$, which can be explained via the geometric interpretation shown in Figure 3. Each hysteresis operator $\delta(\alpha, \beta, u(t))$ is assigned to a point $(\alpha, \beta)$ on the $\alpha-\beta$ plane. All such $(\alpha, \beta)$ points are in a region satisfying $u_{\max } \geq \alpha \geq \beta \geq$, $u_{\text {min }} \geq 0$ which is referred to as the limiting triangle $T_{0}$. According to the values of $\delta(\alpha, \beta, u(t)), T_{0}$ is divided into three regions denoted by $S_{0}, S_{1}$, and $S_{2}$, where $\delta=0, \delta=\mu_{1}$, and $\delta=\mu_{2}$, respectively. The interface between $S_{0}$ and the other two regions is a horizontal line characterized by $\alpha=\max \{u(\tau) \mid 0 \leq \tau \leq t\}=M_{1}=M_{\max }$ in which $M_{\max }$ is the maximum local maximum. The interface between $S_{1}$ and $S_{2}$ is a staircase line with vertex coordinates $\left(m_{i}, M_{j}\right)$, where $i=0,1, \cdots, m$ and $j=1,2, \cdots, n$,

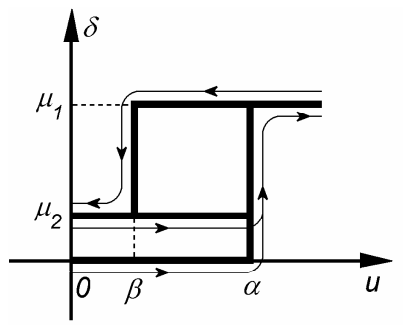

$$
\begin{aligned}
& u \text { - input } \\
& \delta \text { - output of the hysteresis } \\
& \quad \text { operator } \\
& \alpha \text { - up switching value } \\
& \beta \text { - down switching value } \\
& \mu_{1} \text { - upper saturation magnitude } \\
& \mu_{2} \text { - lower saturation magnitude }
\end{aligned}
$$

Figure 2. The novel hysteresis operator $\delta(\alpha, \beta, u(t))$ [3].

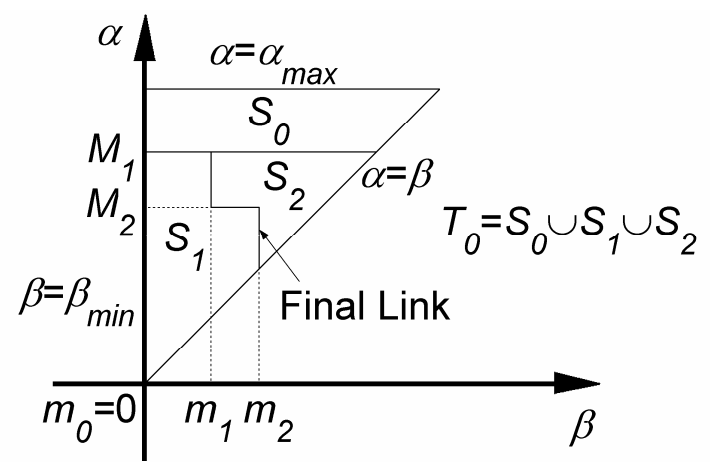

Figure 3. Geometric interpretation of the RI hysteresis model.
$M_{j}$ and $m_{i}$ are the historical local ma- xima and minima of $u(t)$, respectively. The link in the $S_{1}-S_{2}$ interface that attaches to the line $\alpha=\beta$ is referred to as the final link and it represents the influence of the changes in input $u(t)$ to the shapes of $S_{0}, S_{1}$, and $S_{2}$. This link is horizontal and goes up at a speed of $|\dot{u}(t)|$ when $\dot{u}(t)>0$, and is vertical and goes left at a speed of $|\dot{u}(t)|$ when $\dot{u}(t)<0$. Noting that

$$
\begin{array}{r}
\iint_{S_{0}(u(t))} 0 \mathrm{~d} \alpha \mathrm{d} \beta=0, \text { so Equation (1) can be reduced to } \\
f(t)=\iint_{S_{1}} \mu_{1}(\alpha, \beta) \mathrm{d} \alpha \mathrm{d} \beta+\iint_{S_{2}} \mu_{2}(\alpha, \beta) \mathrm{d} \alpha \mathrm{d} \beta
\end{array}
$$

The motion of the final link will wipe out certain vertexes and links, or $\left(m_{i}, M_{j}\right)$ pairs whenever $u(t)<m_{m}$ or $u(t)>M_{n}$. A modified version of the wipe-out property [4] of the CP hysteresis model governs such wipe-out processes. The modification is that in the RI hysteresis model, the first local minimum $m_{0} \equiv 0$ and the maximum local maximum $M_{\max }$ are never wiped out. Besides, the congruency property [4] of the CP hysteresis model still applies to the hysteresis loops but not to the initial ascending curve in the RI hysteresis model as the initial ascending curve is not part of any hysteresis loops.

Equations (1) and (2) involve double integration which is difficult to implement in practice, so an alternative model expression without calculus is desirable and is given in [3]. The rationale behind this alternative model expression is that the $S_{1}$ and $S_{2}$ regions in Equation (2) can be treated as a result of the unification and subtraction of a series of triangular regions $\Delta S_{1}\left(u\left(t_{i}\right), u\left(t_{i+1}\right)\right)$ and $\Delta S_{2}\left(u\left(t_{i}\right), u\left(t_{i+1}\right)\right)$. Then the integration of

$(\alpha, \beta, u(t))$ over each of such triangular regions can be pre-identified and used to calculate $f(t)$ through a series of adding and subtracting operations.

Specifically, each of such triangular regions represents the shape change in $S_{1}$ or $S_{2}$ as the input changes monotonically between two adjacent extrema $u\left(t_{i}\right)$ and $u\left(t_{i+1}\right)$ in the input history which are not wiped-out. The changes in the model output due to $\Delta S_{1}\left(u\left(t_{i}\right), u\left(t_{i+1}\right)\right)$ and $\Delta S_{2}\left(u\left(t_{i}\right), u\left(t_{i+1}\right)\right)$ are represented by the following two functions.

$$
\begin{aligned}
& F_{1}\left(u\left(t_{i}\right), u\left(t_{i+1}\right)\right)=\iiint_{\Delta S_{1}\left(u\left(t_{i}\right), u\left(t_{i+1}\right)\right)} \mu_{1}(\alpha, \beta) \mathrm{d} \alpha \mathrm{d} \beta \\
& F_{2}\left(u\left(t_{i}\right), u\left(t_{i+1}\right)\right)=\iiint_{\Delta S_{1}\left(u\left(t_{i}\right), u\left(t_{i+1}\right)\right)} \mu_{1}(\alpha, \beta) \mathrm{d} \alpha \mathrm{d} \beta
\end{aligned}
$$

Thus, the RI hysteresis model output can be expressed as a linear combination of $F_{1}\left(u\left(t_{i}\right), u\left(t_{i+1}\right)\right)$ and $F_{2}\left(u\left(t_{i}\right), u\left(t_{i+1}\right)\right)$, as follows. Denoting two adjacent local minimum and local maximum by $m$ and $M$, respectively, 
the following relationships related to Equations (3) and (4) are obtain by using the congruency property and the property of the initial ascending curve.

$$
\begin{gathered}
F_{1}(M, m)=F_{1}(m, M) \\
=\iint_{\Delta S_{1}(M, m)} \mu_{1}(\alpha, \beta) \mathrm{d} \alpha \mathrm{d} \beta=f_{1 M}-f_{1 M m} \\
F_{2}(M, m)=\iint_{\Delta S_{2}(M, m)} \mu_{2}(\alpha, \beta) \mathrm{d} \alpha \mathrm{d} \beta \\
=f_{2 M m}-f_{2 M}=f_{2 M m}-0=f_{2 M m}
\end{gathered}
$$

where $f_{j M}(j=1,2)$ represents the part in $f(t)$ contributed by the $S_{j}$ region at the moment when $u(t)$ is initially increased form 0 to $M$; and $f_{j M m}(f j=1,2)$ the part in $f(t)$ contributed by the $S_{j}$ region at the moment when $u(t)$ is subsequent decreased to $m$ from $M$. Assume that the historical local minima and maxima of $u(t)$ that are not wiped out are

$\beta_{\text {min }} \equiv m_{0} \equiv 0<m_{1}<\cdots<m_{n}<M_{n}<\cdots<M_{1} \leq \alpha_{\max }$ during $\dot{u}(t)>0$, and

$\beta_{\min }=m_{0}=0<m_{1}<\cdots<m_{n-1}<M_{n}<\cdots<M_{1} \leq \alpha_{\max } \quad$ during $\dot{u}(t)<0$. Then, if the input monotonically increases, i.e., $\dot{u}(t)>0$, one has

$$
\begin{aligned}
f(t)= & \sum_{k=0}^{n-1}\left[F_{1}\left(M_{k+1}, m_{k}\right)-F_{1}\left(M_{k+1}, m_{k+1}\right)\right] \\
& +F_{1}\left(u(t), m_{n}\right)+\sum_{k=1}^{n-1}\left[F_{2}\left(M_{k}, m_{k}\right)-F_{2}\left(M_{k+1}, m_{k}\right)\right] \\
& +F_{2}\left(M_{n}, m_{n}\right)-F_{2}\left(u(t), m_{n}\right)
\end{aligned}
$$

and if the input monotonically decreases, i.e., $\quad \dot{u}(t)<0$

$$
\begin{aligned}
f(t)= & \sum_{k=0}^{n-2}\left[F_{1}\left(M_{k+1}, m_{k}\right)-F_{1}\left(M_{k+1}, m_{k+1}\right)\right] \\
& +F_{1}\left(M_{n}, m_{n-1}\right)-F_{1}\left(M_{n}, u(t)\right) \\
& +\sum_{k=1}^{n-1}\left[F_{2}\left(M_{k}, m_{k}\right)-F_{2}\left(M_{k+1}, m_{k}\right)\right]+F_{2}\left(M_{n}, u(t)\right)
\end{aligned}
$$

For the ease of parameter identification, define two new functions as

$$
\begin{aligned}
& F\left(M_{\text {max }}\right)=F_{1}\left(M_{\text {max }}, 0\right) \\
& G(M, m)=F_{1}(M, m)-F_{2}(M, m)
\end{aligned}
$$

where $F\left(M_{\max }\right)$ is the model output increment when $u(t)$ is increased from 0 to $M_{\max }$ along the initial ascending curve, and $G(M, m)$ is the model output decrement when $u(t)$ is subsequently decreased to $m$. The values of $F\left(M_{\max }\right)$ and $G(M, m)$ under different $m$, $M$, and $M_{\max }$ are readily measurable from a hysterical plant, so suitable expressions of $F\left(M_{\max }\right)$ and $G(M, m)$ can be found. This is to be described in Section 4.

Finally, Substituting Equations (8) and (9) into Equations (6) and (7) yields the alternative expression of the RI hysteresis model for practical uses. For $\dot{u}(t)>0$

$$
\begin{aligned}
f(t)= & F\left(M_{\max }\right)-\sum_{k=1}^{n-1}\left[G\left(M_{k}, m_{k}\right)-G\left(M_{k+1}, m_{k}\right)\right] \\
& -\left[G\left(M_{n}, m_{n}\right)-G\left(u(t), m_{n}\right)\right]
\end{aligned}
$$

and for $\dot{u}(t)<0$

$$
\begin{aligned}
f(t)= & F\left(M_{\max }\right)-\sum_{k=1}^{n-1}\left[G\left(M_{k}, m_{k}\right)-G\left(M_{k+1}, m_{k}\right)\right] \\
& -G\left(M_{n}, u(t)\right)
\end{aligned}
$$

\section{A Model of Piezoelectric Actuators}

The model of PEA is developed by cascading a vibration sub-model and a creep sub-model to the above RI hysteresis model, as shown in Figure 4. $u(t)$ represents the voltage input, and $x(t)$ represents the displacement of the PEA or the model output.

The vibration sub-model is represented by means of a second order system under the assumption that the mass driven by the PEA is much larger than the mass of the PEA itself [9], i.e.,

$$
2 \zeta \omega_{n} \dot{x}_{1}(t)+\omega_{n}^{2} x_{1}(t)=f(t)
$$

where $\zeta$ and $\omega_{n}$ are the damping ratio and the natural frequency, respectively; and $f(t)$ is the hysteresis being represented by using the RI hysteresis model discussed in the previous section. Since the RI hysteresis $f(t)$ does not introduce phase lag, the phase lag between $x(t)$ and $u(t)$ is resulted from the vibration sub-model (Given the fact that the magnitude of creep is very small, its phase lag can be neglected). Thus, the values of $\zeta$ and $\omega_{n}$ can be estimated by fitting the frequency-phase response of Equation (12) to the measured frequencyphase response of a PEA.

The parameter identification of the RI hysteresis model involves the identification of the functions $F\left(M_{\max }\right)$ and $G(M, m)$. To do this, some input-output data or the values of $f(t)$ corresponding to certain inputs $u(t)$ need to be found. However $f(t)$ is difficult to measure in practice, so in this study such $f(t)$ values is calculated

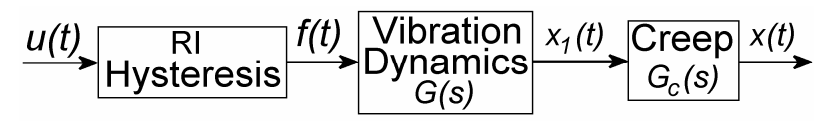

Figure 4. Integrated model of a PEA. 
from Equation (12) by using $x_{1}(t)$ and its derivatives when the PEA is subject to $u(t)$. It should be noted that in this process, creep is again neglected due to its small magnitude and $x(t)$ is taken as $x_{1}(t)$, which is measurable. The derivatives of $x_{1}(t)$ can be approximately found either by firstly passing through the measured $x(t)$ through a low pass filter to suppress the noise and then differentiating the low pass filter output or by a state estimator such as an $\alpha-\beta-\gamma$ filter. Once such $u(t)$ and $f$ ( $t)$ data are obtained, a suitable expressions of $F\left(M_{\max }\right)$ and $G(M, m)$ can then be found with their parameters estimated (to be discussed in Section 4).

The creep in Figure 4 is represented by means of a linear dynamic system model $G_{2}(s)$ taking $x_{1}(t)$ as input and generating a creep displacement $x_{2}(t) . x_{2}(t)$ is then added to $x_{1}(t)$ to obtain the total displacement output of the PEA, $x(t)$, as shown in Figure 5. The form and the parameters of $G_{2}(s)$ can be determined by system identification methods.

Eventually, with a voltage input $u(t)$, a displacement output $x(t)$ in Figure $\mathbf{4}$ can be derived from Equations (10), (11), (12), and

$$
\begin{aligned}
G_{c}(s) & =X(s) / X_{1}(s)=\left[X_{1}(s)+X_{2}(s)\right] / X_{1}(s) \\
& =1+G_{2}(s)
\end{aligned}
$$

where $X_{1}(s), X_{2}(s)$, and $X(s)$ are the Laplace transforms of $x_{1}(t), x_{2}(t)$, and $x(t)$ respectively.

\section{Experiments and Results}

\subsection{Experiment Setup}

Experimental validations of the PEA model are carried out on a PEA (P-753, Physik Instrumente). The actuator can generate motion in a range of $15 \mu \mathrm{m}$ with a resolution of $0.5 \mathrm{~nm}$. For displacement measurements, a builtin capacitive displacement sensor of the P-753 PEA with a resolution of $1 \mathrm{~nm}$ was used. Both the actuator and the sensor are interfaced to a host computer via an I/O board (PCI-DAS1602/16, Measurement Computing Corporation) and controlled by SIMULINK programs. All measured displacements presented in this study were measured with a sampling interval of $0.05 \mathrm{~ms}$.

\subsection{Vibration Sub-Model Parameter Estimation}

As mentioned in the previous section, $\zeta$ and $\omega_{n}$ in Equation (12) were estimated by fitting the phase frequency response of Equation (12) to the measured response of the PEA based on the method of least squares. The phase frequency response curve of the PEA displacements was measured by feeding sinusoidal voltages between 0 and $1000 \mathrm{~Hz}$ to the PEA and then calculating the phase differences between the input voltages and the measured output displacements. The fitted results are shown in Figure 6, from which $\zeta$ and $\omega_{n}$ were estimated as 0.788 and $5352 \mathrm{rad} / \mathrm{s}$, respectively.

\subsection{RI Hysteresis Sub-Model Parameter Estimation}

To identify $F\left(M_{\text {max }}\right)$ and $G(M, m)$ in Equations (8) and (9), the displacements of the PEA were measured under the voltage inputs determined by

$$
u(t)=V_{p p} / 2+\left(V_{p p} / 2\right) \sin (200 \pi t+1.5 \pi)
$$

where $V_{p p}$ is the peak-to-peak magnitudes. Since the frequency of voltage input, i.e., $100 \mathrm{~Hz}$, was high, creep was insignificant over a few periods of the waveforms and thus neglected. The minimum and maximum of the voltage applied to the PEA were set to $0 \mathrm{~V}$ and $70 \mathrm{~V}$, respectively. $V_{p p}$ (or $M_{\text {max }}$ ), $M$ and $m$ were then taken values from $0 \mathrm{~V}$ to $70 \mathrm{~V}$ with a step of $5 \mathrm{~V}$, respectively and thus the series of $u(t)$ were determined. By applying $u(t)$ of different $V_{p p}$ or $M_{\max }$ to the PEA, the displacements of the actuator were measured. Each measured displacement waveform was the $x_{1}(t)$ in Equation (12) corresponding to a given $u(t) . \dot{x}_{1}(t)$ and $\ddot{x}_{1}(t)$ were estimated by an $\alpha-\beta-\gamma$ filter $\left(\alpha=8.7 \times 10^{-2}\right.$, $\left.\beta=3.9 \times 10^{-3}, \gamma=2.4 \times 10^{-3}\right)$ from the measured $x_{1}(t)$. $x_{1}(t), x_{1}(t)$, and $x_{1}(t)$ were then substituted into Equation (12) to calculate the "measured" $f(t)$ corresponding to the given $u(t)$. In the following, the values of $f(t)$ and the corresponding $u(t)$ from the initial conditions of $t=0, u(t)=0$, and $f(t)=0$ were examined. Once $u(t)$ was increased to reach a given $M_{\max }$ from $u(t=0)=0$, the corresponding $f(t)$ value was found and taken as a value of $F\left(M_{\max }\right)$. Similarly, once

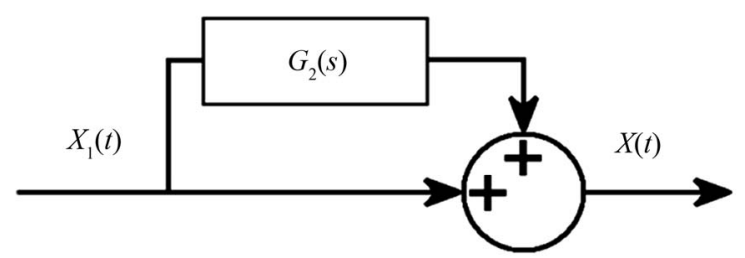

Figure 5. The creep sub-model, i.e., $G_{c}(s)$ in Figure 4.

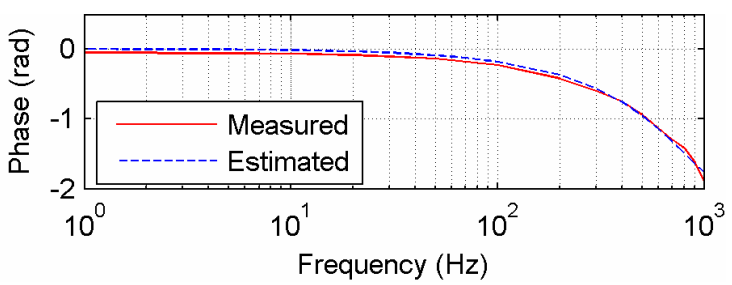

Figure 6. Measured and estimated phase frequency responses. 
the voltage was subsequently decreased from $M=M_{\max }$ to a specific value of $m$, the $f(t)$ value was found and taken again. This $f(t)$ value was subtracted from the $F\left(M_{\max }\right)$ value just measured and the result was taken as a value of $G(M, m)$ at this specific $(M, m)$ point. It was found that the measured points $\left(M_{\max }, F\left(M_{\max }\right)\right)$ resemble a smooth curve, and the measured points $(M, m, G(M, m))$ resemble a smooth surface. Hence $F\left(M_{\max }\right)$ was represented by a polynomial

$$
\begin{aligned}
& F\left(M_{\text {max }}\right) \\
= & b_{0}+b_{1} M_{\text {max }}+b_{2} M_{\text {max }}^{2}+b_{3} M_{\text {max }}^{3}+b_{4} M_{\text {max }}^{4}
\end{aligned}
$$

And $G(M, m)$ was represented by a trend surface

$$
\begin{aligned}
G(M, m)= & p_{1}+p_{2} m+p_{3} M+p_{4} m^{2}+p_{5} M m \\
& +p_{6} M^{2}+p_{7} m^{3}+p_{8} M m^{2}+p_{9} M^{2} m \\
& +p_{10} M^{3}+p_{11} m^{4}+p_{12} M m^{3}+p_{13} M^{2} m^{2} \\
& +p_{14} M^{3} m+p_{15} M^{4}
\end{aligned}
$$

Then the values of the parameters in Equations (15) and (16) were estimated by using the maximum likelihood method based on the measurements of $F\left(M_{\max }\right)$ and $G(M, m)$. The estimated parameter values are shown in Table 1. With these parameters, the RI hysteresis of the actuators can be evaluated for any given input $u(t)$ by using Equations (10) and (11).

\subsection{Creep Sub-Model Parameter Estimation}

Creep is a slow effect. To identify the parameters involved in the creep sub-model, a step voltage input of $30 \mathrm{~V}$ was applied to the PEA for an extended period of time (30 s). The output displacement $x(t)$ of the PEA was measured. And the corresponding $x_{1}(t)$ was obtained by simulation using the identified hysteresis and vibration sub-models. Then $x_{2}(t)$, which is the measured output of $G_{2}(s)$

Table 1. Estimated values of the parameters in Equations (15) and (16).

\begin{tabular}{cccccc}
\hline Par. & Value & Par. & Value & Par. & Value \\
\hline$b_{0}$ & 4.84 & $p_{3}$ & $2.85 \times 10^{6}$ & $p_{10}$ & 362 \\
$b_{1}$ & -745 & $p_{4}$ & $2.26 \times 10^{4}$ & $p_{11}$ & 4.58 \\
$b_{2}$ & $5.50 \times 10^{4}$ & $p_{5}$ & $-3.35 \times 10^{4}$ & $p_{12}$ & -9.67 \\
$b_{3}$ & $2.85 \times 10^{6}$ & $p_{6}$ & $3.01 \times 10^{3}$ & $p_{13}$ & 5.17 \\
$b_{4}$ & $5.75 \times 10^{4}$ & $p_{7}$ & 46.7 & $p_{14}$ & 1.66 \\
$p_{1}$ & $9.10 \times 10^{5}$ & $p_{8}$ & -49.0 & $p_{15}$ & -3.03 \\
$p_{2}$ & $-2.77 \times 10^{6}$ & $p_{9}$ & -178 & & \\
\hline
\end{tabular}

in Figure 5 induced by the input $x_{1}(t)$, was obtained by $x_{2}(t)=x(t)-x_{1}(t)$. By using the System Identification Toolbox in MATLAB, an ARX (Auto Regressive with eXogenous input) model with a sampling period of $0.1 \mathrm{~s}$ and having 4 poles, 1 zero, and 1 sampling period of delay between output and input was identified by the least squares method to model creep. This ARX model was then converted into a continuous-time model as

$$
G_{2}(s)=\frac{0.1804 s^{3}+4.181 s^{2}+47.84 s+9.404}{s^{4}+14.10 s^{3}+610.1 s^{2}+1018 s+125.0}
$$

The simulated (by using Equation (17)) and the measured creep displacements of the PEA are compared in Figure 7.

\subsection{Validation of the Model of PEA}

The input voltage $u(t)$ used for validating the integrated model of PEA is shown in Figure 8. In the experiments, $1 / T_{v}$ was set to $50,100,200,300,400$, and $500 \mathrm{~Hz}$. Two of the measured results are shown in Figure 9; along with the simulation results obtained form the developed model, for the purpose of comparison. The rootmean-square (RMS) errors and the maximum errors calculated over two periods of the $u(t)$ waveform are given in Table 2.

It can be seen from Figure 9 and Table 2 that the PEA model integrating the RI hysteresis model with the vibration and creep sub-models can represent the dynamics of the PEA with good accuracy (the RMS error is less than $1 \%$ of the maximum displacement of the PEA) when the PEA is subject to voltage input signals with a frequency up to $400 \mathrm{~Hz}$. The increase in the RMS error as the input frequency increases is considered as a result of the unmodeled high-frequency dynamics of the PEA. The higher

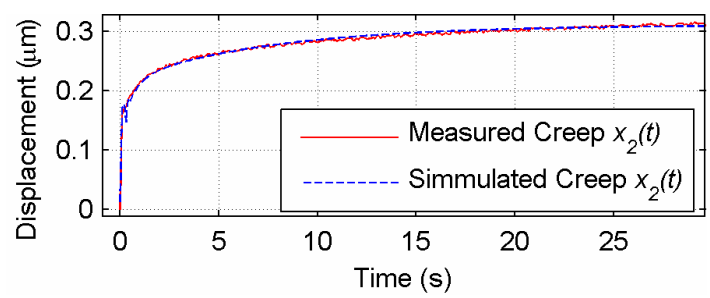

Figure 7. Measured and simulated creep displacements.

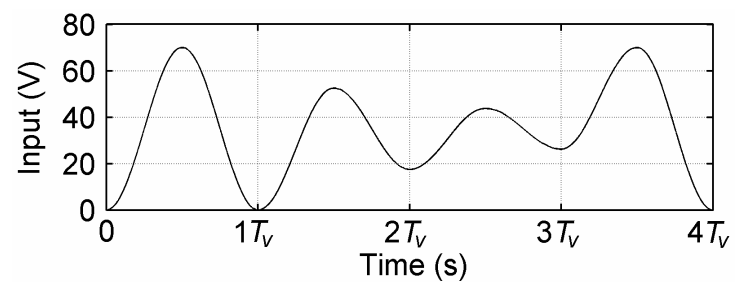

Figure 8. $u(t)$ used for PEA model validation. 

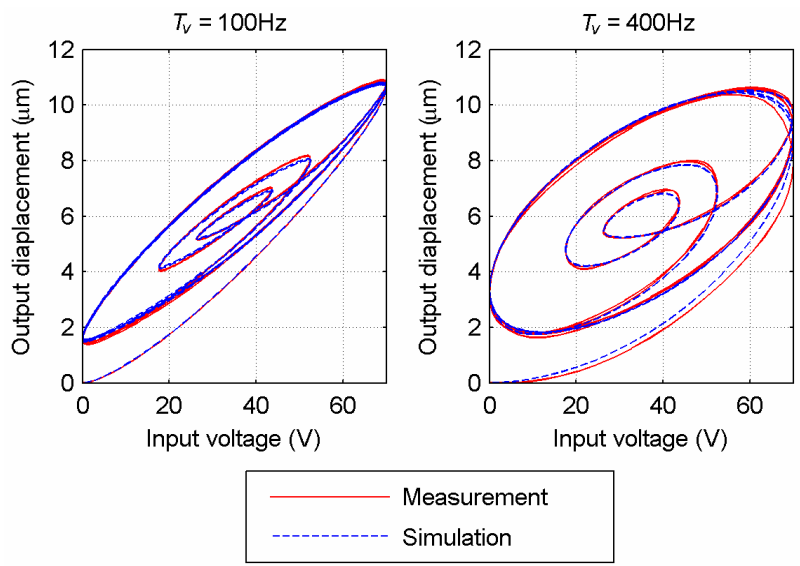

Figure 9. PEA model validation.

Table 2. Errors in the PEA model validation experiments.

\begin{tabular}{ccc}
\hline $1 / T_{v}(\mathrm{~Hz})$ & RMS Error $(\mu \mathrm{m})$ & Maximum Error $(\mu \mathrm{m})$ \\
\hline 50 & 0.093 & 0.212 \\
100 & 0.066 & 0.157 \\
200 & 0.055 & 0.127 \\
300 & 0.059 & 0.136 \\
400 & 0.095 & 0.252 \\
500 & 0.188 & 0.593 \\
\hline
\end{tabular}

RMS errors in the low frequency compared with those at medium are thought to be caused by the $2^{\text {nd }}$-order approximation of the vibration dynamics that leads to a smaller phase lag then reality.

\section{Conclusions}

Accurate models of PEAs are highly desirable for both better understanding the behavior of such actuators and controller design. Such models can usually be constructed by cascading three sub-models together, each representing the RI hysteresis, the vibration dynamics, and the creep effect, respectively. While the vibration dynamics and the creep effect have been accurately modeled in the literature, there are still problems concerning the modeling of RI hysteresis. Traditionally, the CP hysteresis model has been widely used to represent the RI hysteresis in PEAs. However, it is found that the CP hysteresis model is incapable of representing the one-sided hysteresis behavior in PEAs since it cannot represent the initial ascending curve, inducing significant inaccuracy. To solve this problem, in this paper, an integrated model of PEAs was developed based on a new RI hysteresis model which is specifically designed to enable the representation of the one-sided hysteresis behavior. The resultant model of PEAs was validated through experiments. And it is concluded that the resultant model developed is capable of representing the dynamic behaviors of PEAs, including one-sided hysteresis, creep, and vibration dynamics accurately, with an RMS error less than $1 \%$ of the maximum PEA displacement and in operations with frequentcies up to $400 \mathrm{~Hz}$.

\section{Acknowledgements}

Financial support from the Natural Science and Engineering Research Council (NSERC) of Canada to the present study is acknowledged.

\section{References}

[1] S. Devasia, E. Eleftheriou and S. O. R. Moheimani, "A Survey of Control Issues in Nanopositioning," IEEE Transactions on Control Systems Technology, Vol. 15, No. 5, 2007, pp. 802-823. doi:10.1109/TCST.2007.903345

[2] D. Croft, G. Shed and S. Devasia, "Creep, Hysteresis, and Vibration Compensation for Piezoactuators: Atomic Force Microscopy Application," Journal of Dynamic Systems, Measurement, and Control, Vol. 123, No. 1, 2001, pp. 35-43. doi:10.1115/1.1341197

[3] J. Y. Peng and X. B. Chen, "Hysteresis Models Based on a Novel Hysteresis Unit," 2011, Unpublished.

[4] I. Mayergoyz, "Mathematical Models of Hysteresis," Physical Review Letters, Vol. 56, No. 15, 1986, pp. 15181521. doi:10.1103/PhysRevLett.56.1518

[5] P. Ge and M. Jouaneh, "Generalized Preisach Model for Hysteresis Nonlinearity of Piezoceramic Actuators," Precision engineering, Vol. 20, No. 2, 1997, pp. 99-111. doi:10.1016/S0141-6359(97)00014-7

[6] H. Hu and R. Ben-Mrad, "On the Classical Preisach Model for Hysteresis in Piezoceramic Actuators," Mechatronics, Vol. 13, No. 2, 2002, pp. 85-94. doi:10.1016/S0957-4158(01)00043-5

[7] G. Song, J. Zhao, X. Zhou and J. A. De Abreu-García, "Tracking Control of a Piezoceramic Actuator with Hysteresis Compensation Using Inverse Preisach Model," IEEE/ASME Transactions on Mechatronics, Vol. 10, No. 2, 2005, pp. 198-209. doi:10.1109/TMECH.2005.844708

[8] X. Yang, W. Li, Y. Wang and G. Ye, "Modeling Hysteresis in Piezo Actuator Based on Neural Networks," Lecture Notes in Computer Science, Vol. 5370, 2008, pp. 290-296. doi:10.1007/978-3-540-92137-0 32

[9] X. B. Chen, Q. Zhang, D. Kang and W. Zhang, "On the Dynamics of Piezoactuated Positioning Systems," Review of Scientific Instruments, Vol. 79, No. 11, 2008, pp. 1161011 to 116101-3. 